\title{
In vivo 3-dimensional Kinematics of Cubitus Valgus after Non-united Lateral Humeral Condyle Fracture
}

\author{
Eugene Kim, Se-Jin Park ${ }^{\circledR}$, Ho-Seok Lee, Jai-Hyung Park, Jong Kuen Park, Sang Hoon Ha, Tsuyoshi Murase ${ }^{1}$, \\ Kazuomi Sugamoto ${ }^{1}$
}

Department of Orthopaedic Surgery, Kangbuk Samsung Hospital, Sungkyunkwan University School of Medicine, Seoul, Korea, ${ }^{1}$ Department of Orthopaedic Surgery, Osaka University Graduate School of Medicine, Osaka, Japan

Background: Nonunion of lateral humeral condyle fracture causes cubitus valgus deformity. Although corrective osteotomy or osteosynthesis can be considered, there are controversies regarding its treatment. To evaluate elbow joint biomechanics in non-united lateral humeral condyle fractures, we analyzed the motion of elbow joint and pseudo-joint via in vivo three-dimensional (3D) kinematics, using 3D images obtained by computed tomography (CT) scan.

Methods: Eight non-united lateral humeral condyle fractures with cubitus valgus and 8 normal elbows were evaluated in this study. CT scan was performed at 3 different elbow positions (full flexion, $90^{\circ}$ flexion and full extension). With bone surface model, 3D elbow motion was reconstructed. We calculated the axis of rotation in both the normal and non-united joints, as well as the rotational movement of the ulno-humeral joint and pseudo-joint of non-united lateral condyle in 3D space from full extension to full flexion.

Results: Ulno-humeral joint moved to the varus on the coronal plane during flexion, $25.45^{\circ}$ in the non-united cubitus valgus group and $-2.03^{\circ}$ in normal group, with statistically significant difference. Moreover, it moved to rotate externally on the axial plane $-26.75^{\circ}$ in the non-united cubitus valgus group and $-3.09^{\circ}$ in the normal group, with statistical significance. Movement of the pseudo-joint of fragment of lateral condyle showed irregular pattern.

Conclusions: The non-united cubitus valgus group moved to the varus with external rotation during elbow flexion. The pseudo-joint showed a diverse and irregular motion. In vivo 3D motion analysis for the non-united cubitus valgus could be helpful to evaluate its kinematics.

(Clin Shoulder Elbow 2018;21(3):151-157)

Key Words: Three-dimensional image; In vivo; Biomechanical phenomena; Elbow joint; Cubitus valgus

\section{Introduction}

Three-dimensional (3D) image data of the human body can provide a great deal of information. Hence, 3D-simulated navigation surgery has been gaining popularity in total knee arthroplasty. Moreover, the elbow joint motion has been introduced by a variety of techniques. ${ }^{1-3)}$ However, a few studies have reported the use of 3D imaging in the upper extremity, including the hand and elbow. ${ }^{4,5)}$
In general, if patients with nonunion of the lateral humeral condyle fracture have no symptoms, there is no need for surgical treatment. However, nonunion of the lateral humeral condyle fracture combined with cubitus valgus deformity and instability often results in pain in the elbow, along with ulnar neuropathy and cosmetic problems. ${ }^{6-9)}$ Treatment for these patients remains controversial. There are operative methods, such as corrective osteotomy and osteosynthesis or distraction osteogenesis and IIizarov frame fixation for correcting valgus deformity. ${ }^{10)}$ However,

Received May 31, 2018. Revised July 26, 2018. Accepted July 27, 2018.

Correspondence to: Se-Jin Park

Department of Orthopaedic Surgery, Kangbuk Samsung Hospital, Sungkyunkwan University School of Medicine, 29 Saemunan-ro, Jongno-gu, Seoul 03181, Korea

Tel: +82-2-2001-2168, Fax: +82-2-2001-2176, E-mail: qortn97@naver.com, ORCID: https://orcid.org/0000-0002-3941-1857

IRB approval: Kangbuk Samsung Hospital (No. KBC12114).

Financial support: This work was supported by the Medical Research Funds from Kangbuk Samsung Hospital. Conflict of interests: None. 
these treatments are not physiologic, with a high rate of complications, such as avascular necrosis, and stiffness. ${ }^{11-14)}$ Herein, we attempted to analyze the motion of the elbow and pseudo-joint of a non-united lateral humeral condyle.

Existing literature of elbow joint movement are derived from various research methods, including simple radiographs, computed tomography $(\mathrm{CT})$, and magnetic resonance imaging scans from cadavers. However, in vivo analysis of kinematics is scarce, as most of these previous studies have been carried out in vitro. We introduce in vivo analysis by 3D images obtained by CT scans of the normal group and non-united cubitus valgus group. In addition, we evaluate 3D kinematics, using computer simulation. With the results, we want to evaluate elbow joint biomechanics in non-united lateral humeral condyle fracture and can help to elucidate appropriate treatments with biomechanical consideration.

\section{Methods}

This study was approved by the Institutional Review Board of Kangbuk Samsung Hospital (No. KBC12114). From November 2011 to May 2018, we retrospectively studied 8 elbows with nonunited lateral humeral condyle fracture and 8 normal elbows.

The average normal carrying angle was $11^{\circ}$ for adult males and $14^{\circ}$ for adult females. ${ }^{15)}$ Cubitus valgus was defined as when the side-to-side difference of the carrying angle between the normal and non-united lateral humeral condyle fracture, as measured by a goniometer, was greater than $15^{\circ} .{ }^{16)}$ We divided the subjects into two groups: the normal group and the nonunited cubitus valgus group (Fig. 1). The normal group was composed of five males and three females. The non-united cubitus valgus group consisted of five males and three females.

In all cases of non-united cubitus valgus, we measured the carrying angle on plane radiograph. Elbows of all cases were scanned by a low-dose CT scan (Light Speed Ultra 16; General Electric, Waukesha, WI, USA) acquired using a 0.5-second scan time, 10-mA tube current, and 120-kV tube voltage. CT scans were performed at three different degrees of elbow position (full flexion, $90^{\circ}$ flexion and full extension). After the recorded images were converted to Digital Imaging and Communications in Medicine data, we used a computer simulation program (Bone Viewer ${ }^{\mathrm{TM}}$; Orthree Co. Ltd., Osaka, Japan) to construct 3D surface models of the humerus, ulna, radius, and non-united fragment of lateral condyle. By superimposing the total elbow of three positions of humerus based on semiautomatic markerless surface-based registration technique using a computer program (Bone Simulator $^{\mathrm{TM}}$; Orthree Co. Ltd.), 3D motion was reconstructed.
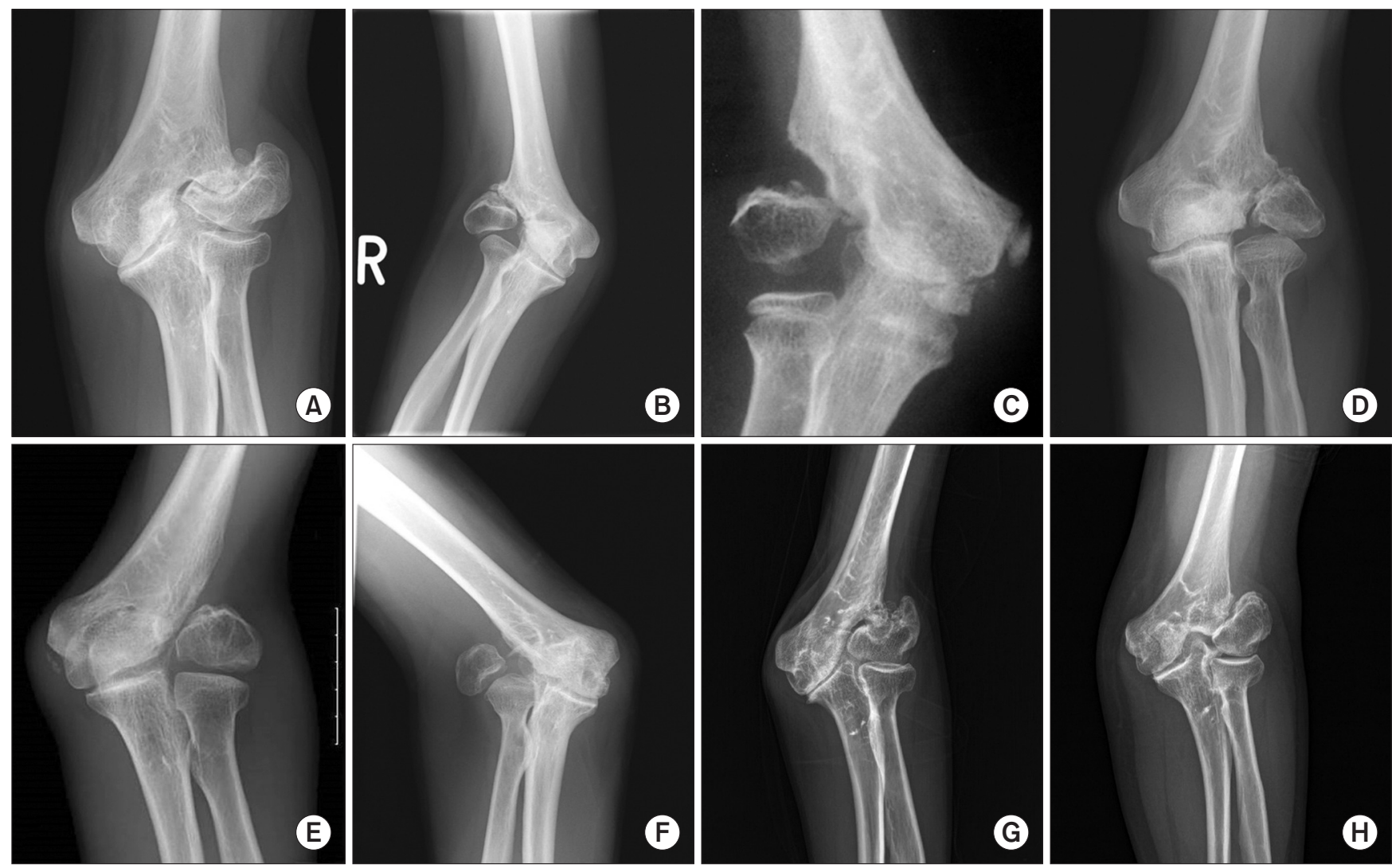

Fig. 1. (A-H) Radiographs of all cases of non-united lateral condyle humerus fracture. 
First, screw axis, which is determined in terms of rotation around and translation along one unique axis, i.e., the 3D deformity axis, using the screw displacement axis technique, was calculated during flexion, and we defined it as the axis of rotation (AOR). ${ }^{4,5}$ Movements of AOR during flexion in groups were compared. We then calculated the rotational axis of the ulno-humeral joint in the position from full extension to full flexion. Based on the calculation, we analyzed the motion of the rotational axis of ulno-humeral joint of elbow and pseudo-joint of affected humerus and movement of the lateral condylar fragment.

In this study, in order to analyze the 3D movements, we used the reference coordinates as defined by the International Society of Biomechanics. ${ }^{17)}$ First, the Y-axis was the center axis, calculated from the moment of inertia of the humerus. The $Z$ axis was defined as the axis at which passes the $Y$ axis and most prominent point of the medial condyle. Moreover, the axis perpendicular to both the $\mathrm{Y}$ - and $\mathrm{Z}$-axes was defined as the $\mathrm{X}$-axis (Fig. 2).

Then, we calculated the rotational movement of the ulna relative to the humerus using the anatomical coordinate system defined above. We prescribed the rotational values for varus $(+) /$ valgus (-) around the $\mathrm{X}$-axis in the coronal plane (YZ), internal $(+) /$ external (-) rotation around the Y-axis in the axial plane (XZ), and flexion (+)/extension (-) around the Z-axis in the sagittal plane $(\mathrm{XY})$ in the Euler angle space, respectively.

\section{Statistical Methods}

All statistical analyses were performed using IBM SPSS ver. 24.0 (IBM Co., Armonk, NY, USA), and values of $p<0.05$ were considered statistically significant. The data about $3 \mathrm{D}$ rotational movement were indicated as the median (interquartile range). The data of two groups were compared using the Mann-Whitney U-test.

\section{Results}

The average age of subjects was 41.50 years (30.25-50.50 years) in the normal group and 54.00 years (41.50-61.75 years) in the non-united cubitus valgus group. In the latter, 3 cases involved the dominant side of the elbow. The median of age at injury was 7.00 years. The median time from injury to assessment was 49.00 years (Table 1 ).

In the non-united cubitus valgus group, the carrying angle was $29.50^{\circ}\left(22.00^{\circ}-33.75^{\circ}\right)$.

Ulno-humeral joints of non-united cubitus valgus group showed significant instability compared with the normal group (Table 2). They moved the varus $\left(25.45^{\circ}\left[21.75^{\circ}\right.\right.$ to $\left.28.08^{\circ}\right]$ vs. $-2.03^{\circ}\left[-3.33^{\circ}\right.$ to $\left.\left.2.20^{\circ}\right], p=0.001\right)$ in the coronal plane with external rotation $\left(-26.75^{\circ}\left[-34.03^{\circ}\right.\right.$ to $\left.-19.48^{\circ}\right]$ vs. $-3.09^{\circ}\left[-4.95^{\circ}\right.$ to $\left.\left.3.95^{\circ}\right], p=0.001\right)$ in the axial plane during flexion. On the other hand, the rotational axis of the normal elbow showed minimal changes during flexion like the hinge joint. While there was little movement of AOR during flexion in the normal elbow, shifting of AOR, which suggests instability, was observed in the nonunited cubitus valgus group (Fig. 3).

Table 1. Demographics for Subjects

\begin{tabular}{lcc}
\hline \multicolumn{1}{c}{ Variable } & Normal group & $\begin{array}{c}\text { Non-united cubitus } \\
\text { valgus group }\end{array}$ \\
\hline No. of patients & 8 & 8 \\
\hline Gender (male:female) & $5: 3$ & $5: 3$ \\
\hline Age (yr) & $41.50(30.25-50.50)$ & $54.00(41.50-61.75)$ \\
Dominant side involvement & - & $3(37.5)$ \\
\hline Age at injury (yr) & - & $7.00(6.00-17.25)$ \\
Time from injury to & - & $49.00(32.75-62.00)$ \\
\hline assessment (yr) & & \\
\hline
\end{tabular}

Values are presented as number only, median (interquartile range), or number (\%).
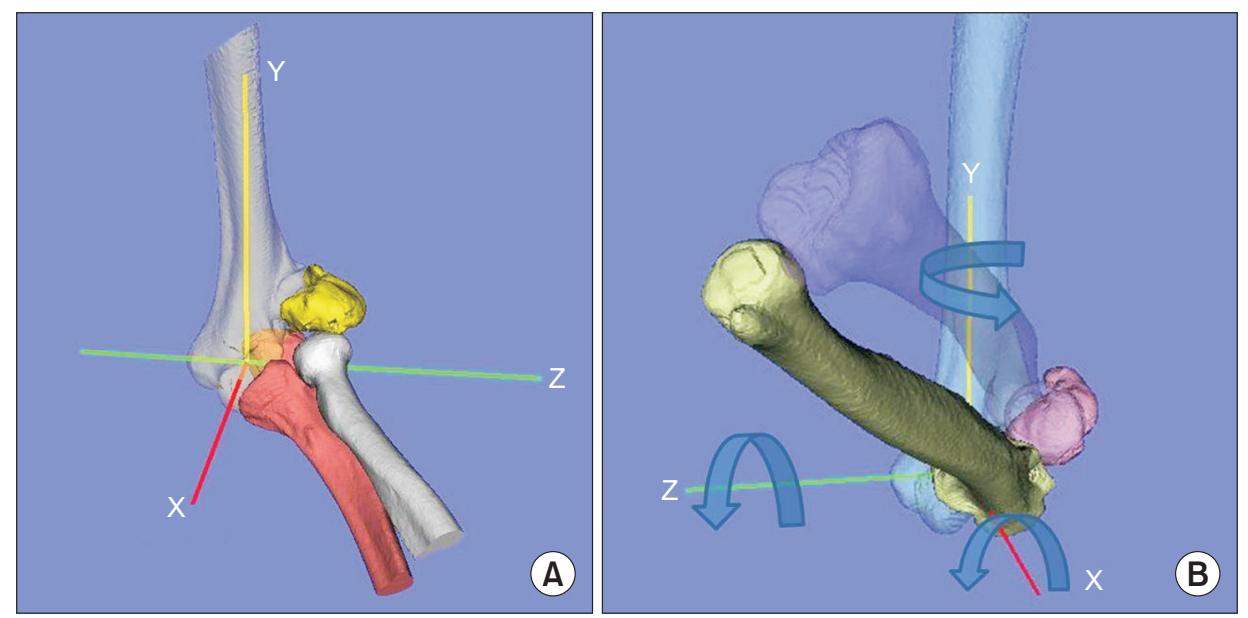

Fig. 2. (A) Anatomic coordinate system of three-dimensional motion for non-united elbow joint in Euler angle space according to International Society of Biomechanics. (B) The rotational movement of non-united elbow joint in Euler angle space, according to the anatomical coordinate system. Rotational values for varus (+)/valgus (-) around the $\mathrm{X}$-axis on the coronal plane (YZ), internal (+)/external (-) rotation around the $\mathrm{Y}$-axis on the axial plane (XZ), and flexion $(+) /$ extension (-) around the $\mathrm{Z}$-axis on the sagittal plane (XY). 
Clinics in Shoulder and Elbow

Vol. 21, No. 3, September, 2018

Table 2. Data of 3-dimensional Rotational Movement of Ulno-humeral Joint of Two Groups

\begin{tabular}{|c|c|c|c|c|c|}
\hline Patient No. & Side & Carrying angle $\left(^{\circ}\right)$ & $\begin{array}{l}\mathrm{X}(\text { varus }[+] / \\
\text { valgus }[-])\left(^{\circ}\right)\end{array}$ & $\mathrm{Y}(\operatorname{IR}[+] / \operatorname{ER}[-])\left(^{\circ}\right)$ & $\begin{array}{c}\mathrm{Z} \text { (flexion }[+] / \\
\text { extension }[-])\left(^{\circ}\right)\end{array}$ \\
\hline \multicolumn{6}{|c|}{ Non-united cubitus valgus group } \\
\hline 1 & Left & 21 & 21.3 & -31.4 & 106.1 \\
\hline 2 & Right & 33 & 26.6 & -34.9 & 109.7 \\
\hline 3 & Right & 27 & 27.7 & -18.1 & 134 \\
\hline 4 & Left & 19 & 23.1 & -17.3 & 97.4 \\
\hline 5 & Left & 25 & 45.9 & -23.6 & 110 \\
\hline 6 & Right & 41 & 24.3 & -51.3 & 76.3 \\
\hline 7 & Left & 32 & 18.2 & -24.2 & 128.2 \\
\hline 8 & Left & 34 & 28.2 & -29.3 & 122.4 \\
\hline Median (IQR) & & $29.50(22.00-33.75)$ & $25.45(21.75-28.08)$ & $-26.75(-34.03--19.48)$ & $109.85(99.58-126.75)$ \\
\hline \multicolumn{6}{|l|}{ Normal group } \\
\hline 1 & Right & & -2.8 & -5.9 & 148.8 \\
\hline 2 & Left & & -3.5 & -3.4 & 139.3 \\
\hline 3 & Right & & 2.3 & 3.5 & 142.7 \\
\hline 4 & Left & & -4.5 & -5.2 & 143.2 \\
\hline 5 & Right & & 2.5 & -4.2 & 133.5 \\
\hline 6 & Right & & 1.9 & 4.5 & 141.3 \\
\hline 7 & Right & & -1.9 & 4.1 & 138.5 \\
\hline 8 & Left & & -2.2 & -2.8 & 143.6 \\
\hline Median (IQR) & & & $-2.03(-3.33-2.20)$ & $-3.09(-4.95-3.95)$ & $142.00(138.70-143.46)$ \\
\hline$p$-value & & & 0.001 & 0.001 & 0.001 \\
\hline
\end{tabular}

IR: internal rotation, ER: external rotation, IQR: interquartile range.
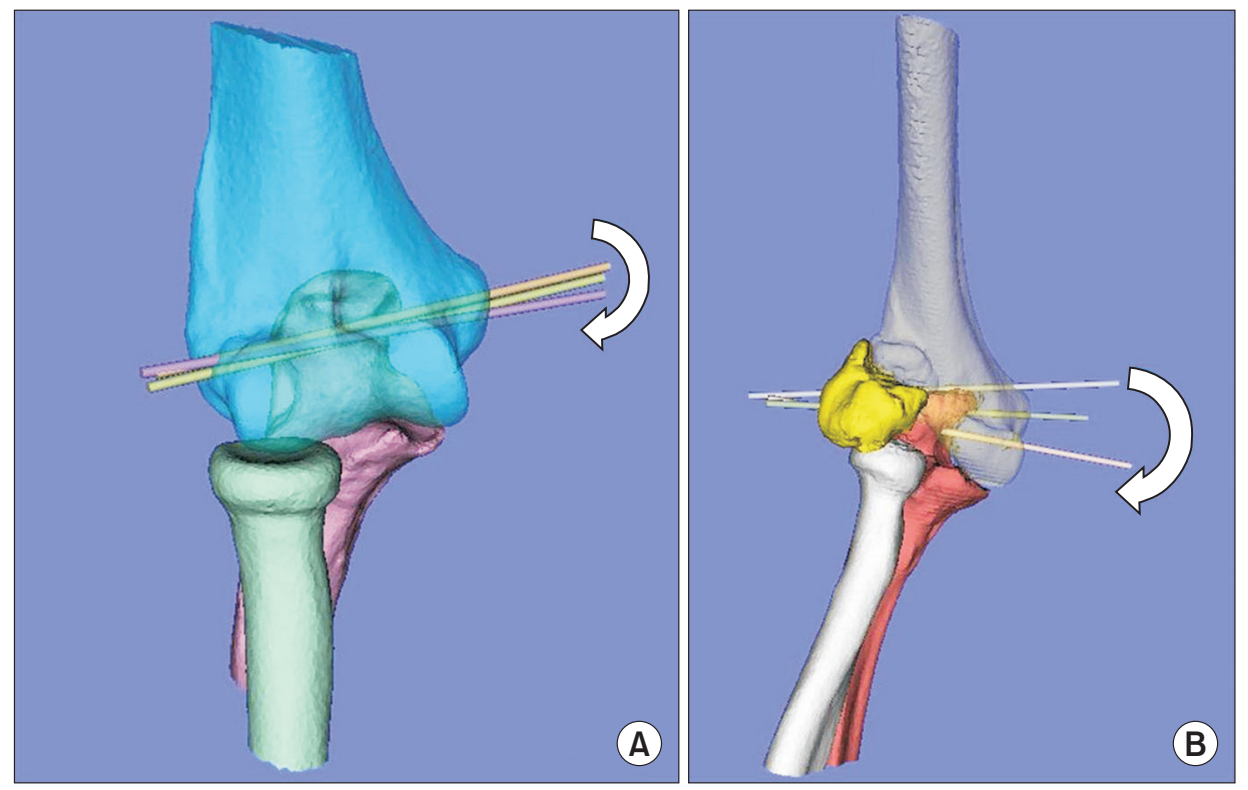

Fig. 3. The movement of axis of rotation (AOR). The normal elbow joint $(\mathrm{A})$ and the elbow joint of the cubitus valgus during full extension to full flexion (B). Arrows indicate the direction of movement of the AOR in full extension, $90^{\circ}$ flexion, and full flexion. 
Table 3. Data of 3-dimensional Rotational Movement of Pseudo-joint of Non-united Lateral Condyle of Humerus

\begin{tabular}{llccr}
\hline Patient No. & Side & X (varus $[+] /$ valgus $[-])\left({ }^{\circ}\right)$ & Y (IR [+]/ER [-]) $\left(^{\circ}\right)$ & Z (flexion $[+] /$ extension $[-])\left({ }^{\circ}\right)$ \\
\hline 1 & Left & 25 & 5.9 & 17.3 \\
\hline 2 & Right & 9.2 & -15.6 & 22.6 \\
\hline 3 & Right & 1 & -1.9 & -9.8 \\
\hline 4 & Left & 19.2 & 15.2 & -4.8 \\
\hline 5 & Left & 7.4 & -24.6 & 59.9 \\
\hline 7 & Right & -0.1 & 34.6 & 53.9 \\
8 & Left & 15.2 & -32.2 & -26.5 \\
Median (IQR) & Left & -15.4 & -23.2 & 32.9 \\
\hline
\end{tabular}

ER: external rotation, IR: internal rotation, IQR: interquartile range.

Movement of the lateral condyle in pseudo-joint varies from case to case (Table 3). In two cases, the lateral condyle moved along a similar course as the ulno-humeral joint in 3D plane (varus-external rotation-flexion; Table 3, patient No. 2, 5). In three cases, they extended during flexion (Table 3, patient No. 3, $4,7)$. In three cases lateral condyles rotated internally, opposite to ulno-humeral joint (Table 3, patient No. 1, 4, 6). One case showed little or no movement (Table 3, patient No. 3).

\section{Discussion}

Non-united lateral humeral condyle fracture may cause cubitus valgus deformity, pain, apprehension, or ulnar neuropathy. These symptoms are possibly triggered during elbow flexion. ${ }^{18)}$ One of the choices that could treat this valgus deformity is corrective osteotomy with osteosynthesis. However, there are various complications associated with this treatment, including failure of bony union, limitation of range of motion, and avascular necrosis. The treatment for these patients remains controversial. Moorehead ${ }^{19)}$ did not treat a case of old lateral condyle fracture on a 17-year-old patient due to tolerable pain and acceptable range of motion. Masada et al. ${ }^{20)}$ reported 30 cases of those who were treated by surgery. After osteosynthesis, pain and apprehension disappeared; however, the range of motion of the elbow decreased. The author stated that osteosynthesis is required for the treatment of non-united lateral humeral condyle fracture only if a patient has serious symptoms in the elbow. They also reported that the results were better in children than in adults. However, Toh et al. ${ }^{11)}$ reported 19 elbows in 18 patients that were divided into two groups on the basis of size of the fragment and location of the fracture line. He analyzed the type of symptoms, carrying angle, range of motion, and functional score. In the case of Milch Type-I injury (non-united fragment transverses lateral to the capitello-trochlear groove), patients showed more frequent symptoms, restricted range of motion, and low func- tional score, compared with Milch Type-II injury (non-united fragment passes through the capitello-trochlear groove). The author insisted that most cases of Milch Type-I injury are symptomatic and surgery should be considered. Miyake et al. ${ }^{21)}$ reported 10 patients who were treated with osteosynthesis; eight patients of Milch Type-II injury achieved osseus union, while 2 patients of Milch Type-I injury showed nonunion or delayed union. In 9 of 10 cases, the total arc of motion was reduced by an average of $20^{\circ}$. In that study, they stated that osteosynthesis is indicated for the treatment in Milch Type-II (non-united fragment passes through the capitello-trochlear groove) long-lasting nonunion of the lateral humeral condyle associated with pain. Gong et al. ${ }^{22)}$ reported 11 cases who underwent surgical treatment. Pain, ulnar neuritis, and functional outcomes improved significantly. However, the combined range of motion decreased by a mean of $11.4^{\circ}$. Here, we recommend osteosynthesis to treat chronic neglected lateral condyle humeral fracture, especially when patients reveal severe pain, instability, ulnar nerve symptoms, and poor functional score. The gold standard of treatment of nonunited lateral humeral condyle fracture remains unclear.

The severity of cubitus valgus can be obtained by measuring the carrying angle. Conventionally, the carrying angle was estimated two-dimensionally on a plain radiograph. ${ }^{1)}$ In a previous study of authors, ${ }^{16)} 3 \mathrm{D}$ carrying angle of 25 normal elbow joints were evaluated using the CT scans. The calculated 3D carrying angle was $20.7^{\circ}$, which was slightly greater than the conventional two-dimensional carrying angle. Despite our efforts to clarify the true carrying angle, the result was derived from static circumstance, without considering the motion of the elbow joint. This study is meaningful for figuring out the carrying angle in vivo from dynamic perspectives. Moreover, in a dynamic situation, the 3D carrying angle could be compared between the normal and deformed elbow joint group.

In a simulation study, the rotational axis of normal elbows demonstrated a hinge joint movement with minor movement 
of AOR. This is compatible with previous studies about normal elbow joint motion. ${ }^{23,24)}$ In cases of non-union, AOR moves during flexion. Ulna moves to varus and external rotation direction, toward the humerus, resulting in instability and potential destruction of the joint surface, as also shown in previous studies. $^{25,26)}$ It is known that the ulnar neuropathy is related to the dynamic structural changes along with the elbow flexion. ${ }^{27,28)}$ External rotated ulna could rub the humerus on the elbow joint, leading to cartilage defect like osteoarthritis. Likewise, symptoms of non-united lateral condyle humerus fracture should be interpreted from a kinematic point of view. The pseudo-joint consisted of non-united fragment and moved in various and irregular directions during elbow flexion. Non-united fragment of lateral condyle was probably influenced by the radial collateral ligament and lateral ulnar collateral ligament. Four cases in which the pseudo-joint rotated externally in the axial plane (Table 3, patient No. 2, 5, 7, 8) were deformed severely to the cubitus valgus compared with other cases. It has been difficult to find any regularity of the pseudo-joint motion to date; Further research containing more subjects will be necessary to analyze and categorize the motion of pseudo-joint with 3D simulation. Moreover, such a study can provide significant information to treat non-united lateral condyle humerus fracture.

From the simulation results, we were able to visualize the 3D motion of normal and non-united elbow joint, analyze motion of ulno-humeral joint and pseudo-joint, during flexion. We confirmed a positive consideration of osteosynthesis of the nonunited bony fragment based on the motion analysis of the bony fragment, not only for corrective osteotomy for correction of the valgus deformity, but also for reconstruction of stable elbows. Furthermore, 3D pseudo-joint motion can help to decide the surgical technique or determine the role as a template in corrective osteotomy. ${ }^{29)}$

This study has several limitations. First, we measured the elbow motion under just 3 sequential static postures. It does not reflect all real dynamic kinematics. Intermediate action and position of elbow motion might present some errors, because they were reconstructed by interpolation of 3 images. Second, the elbow is not only a hinge joint, but also a trochoid joint. We focused on the ulno-humeral joint, which can just bend and stretch. The non-united lateral condyle fragment adjoins the radial head, which can rotate toward the ulna. It would be insufficient to analyze the pseudo-joint motion. Third, although we used a lower radiation dose than that used in routine diagnostic $\mathrm{CT}$, there was a risk of radiation exposure. A previous experimental study showed that the radiation exposure required by this system is one thirtieth that of the normal radiation dose associated with a conventional diagnostic CT scan with similar accuracy. ${ }^{30)}$ Fourth, our database is still small. Although the pseudojoint showed various motions from case to case during elbow flexion, it was difficult to find regularity.
Further studies comparing 3D kinematics and severity of symptoms of the affected elbow joint can provide more information to explain how cubitus valgus and elbow instability can lead to typical symptoms. In addition to the fracture type, symptom severity, cosmetic issue, as well as 3D kinematic viewpoint of ulno-humeral and pseudo-joint would be reflected in consideration of surgical indication, by following researches. We expect that 3D kinematics will be referred not only to decide whether or not to perform surgery, but also to select the surgical technique based on the biomechanical behavior of the bony fragment. However, with this study, we suggest that osteosynthesis should not be considered with non-united fracture with good elbow range of motion.

\section{Conclusion}

The 3D computed simulation system provides important information about the 3D deformity and 3D kinematics of elbow motion. As is known in this study, the normal elbow joints showed nearly a hinge-joint motion during its flexion, while ulno-humeral joint in the non-united elbow joints showed instability without fixed AOR. Ulna rotated to varus direction in the coronal plane and rotated externally in the axial plane. Pseudo-joint showed diverse and irregular motion. A study via $3 \mathrm{D}$ real dynamic kinematics is needed to analyze the symptom development according to the location of non-united fragment in patients with specific symptoms and to consider surgical treatment accordingly. Based on this study, we confirmed the possibility that $3 \mathrm{D}$ simulation could give more information and be an indicator of treatment of non-united lateral condyle humerus fracture.

\section{References}

1. Morrey BF, Chao EY. Passive motion of the elbow joint. J Bone Joint Surg Am. 1976;58(4):501-8.

2. McDonald CP, Moutzouros V, Bey MJ. Measuring dynamic invivo elbow kinematics: description of technique and estimation of accuracy. J Biomech Eng. 2012;134(12):124502.

3. Bottlang M, Madey SM, Steyers CM, Marsh JL, Brown TD. Assessment of elbow joint kinematics in passive motion by electromagnetic motion tracking. J Orthop Res. 2000;18(2):195202.

4. Kim E, Park SJ, Jeong HJ, et al. In vivo 3D kinematics of axis of rotation in malunited monteggia fracture dislocation. Clin Shoulder Elbow. 2014;17(1):25-30.

5. Kim E, Moritomo H, Murase T, Masatomi T, Miyake J, Sugamoto $\mathrm{K}$. Three-dimensional analysis of acute plastic bowing deformity of ulna in radial head dislocation or radial shaft fracture using a computerized simulation system. J Shoulder Elbow Surg. 2012;21(12):1644-50. 
6. Eamsobhana P, Kaewpornsawan K. Should we repair nonunion of the lateral humeral condyle in children? Int Orthop. 2015;39(8):1579-85.

7. Toh S, Tsubo K, Nishikawa S, Inoue S, Nakamura R, Harata $\mathrm{S}$. Long-standing nonunion of fractures of the lateral humeral condyle. J Bone Joint Surg Am. 2002;84(4):593-8.

8. Smith FM. An eighty-four year follow-up on a patient with ununited fracture of the lateral condyle of the humerus. A case report. J Bone Joint Surg Am. 1973;55(2):378-80.

9. Kalenak A. Ununited fracture of the lateral condyle of the humerus. A 50 year follow-up. Clin Orthop Relat Res. 1977;(124):181-3.

10. Piskin A, Tomak Y, Sen C, Tomak L. The management of cubitus varus and valgus using the llizarov method. J Bone Joint Surg Br. 2007;89(12):1615-9.

11. Toh S, Tsubo K, Nishikawa S, Inoue S, Nakamura R, Narita S. Osteosynthesis for nonunion of the lateral humeral condyle. Clin Orthop Relat Res. 2002;(405):230-41.

12. Shimada K, Masada K, Tada K, Yamamoto T. Osteosynthesis for the treatment of non-union of the lateral humeral condyle in children. J Bone Joint Surg Am. 1997;79(2):234-40.

13. Agarwal A, Qureshi NA, Gupta N, Verma I, Pandey DK. Management of neglected lateral condyle fractures of humerus in children: a retrospective study. Indian J Orthop. 2012;46(6):698-704.

14. Papandrea R, Waters PM. Posttraumatic reconstruction of the elbow in the pediatric patient. Clin Orthop Relat Res. 2000;(370):115-26.

15. Amis AA, Miller JH. The elbow. Clin Rheum Dis. 1982;8(3): 571-93.

16. Park S, Kim E. Estimation of carrying angle based on CT images in preoperative surgical planning for cubitus deformities. Acta Med Okayama. 2009;63(6):359-65.

17. Wu G, van der Helm FC, Veeger HE, et al. ISB recommendation on definitions of joint coordinate systems of various joints for the reporting of human joint motion--part II: shoulder, elbow, wrist and hand. J Biomech. 2005;38(5):981-92.

18. Vanderpool DW, Chalmers J, Lamb DW, Whiston TB. Peripheral compression lesions of the ulnar nerve. J Bone Joint Surg Br. 1968;50(4):792-803.
19. Moorehead EL. Old untreated fracture of external condyle of humerus. Factors influencing choice of treatment. Surg Clin. 1919;3:987-9.

20. Masada K, Kawai H, Kawabata H, Masatomi T, Tsuyuguchi $\mathrm{Y}$, Yamamoto K. Osteosynthesis for old, established non-union of the lateral condyle of the humerus. J Bone Joint Surg Am. 1990;72(1):32-40.

21. Miyake J, Shimada K, Masatomi T. Osteosynthesis for longstanding nonunion of the lateral humeral condyle in adults. J Shoulder Elbow Surg. 2010;19(7):958-64.

22. Gong MQ, Huang XW, Wang C, et al. Management of chronic neglected lateral condyle elbow non-union in adults: functional results of a cohort study and a proposed treatment algorithm. Int Orthop. 2017;41(1):157-64.

23. London JT. Kinematics of the elbow. J Bone Joint Surg Am. 1981;63(4):529-35.

24. Tanaka S, An KN, Morrey BF. Kinematics and laxity of ulnohumeral joint under valgus-varus stress. J Musculoskelet Res. 1998;2(1):45-54.

25. Horn BD, Herman MJ, Crisci K, Pizzutillo PD, MacEwen GD. Fractures of the lateral humeral condyle: role of the cartilage hinge in fracture stability. J Pediatr Orthop. 2002;22(1):8-11.

26. McKee MD. Displaced fractures of the lateral epicondyle of the humerus and posterolateral rotatory instability of the elbow. J Bone Joint Surg Am. 2003;85(6):1165-6; author reply 1166.

27. Bozentka DJ. Cubital tunnel syndrome pathophysiology. Clin Orthop Relat Res. 1998;(351):90-4.

28. Ochi K, Horiuchi Y, Morisue H, Harato K, Tanikawa H, Okubo M. Association between the disease severity and extraneural pressure induced by maximum elbow flexion in patients with cubital tunnel syndrome. J Plast Surg Hand Surg. 2013;47(3):219-23.

29. Murase T, Oka K, Moritomo H, Goto A, Yoshikawa H, Sugamoto $\mathrm{K}$. Three-dimensional corrective osteotomy of malunited fractures of the upper extremity with use of a computer simulation system. J Bone Joint Surg Am. 2008;90(11):2375-89.

30. Kim E, Lee S, Jeong HJ, et al. Three-dimensional scapular dyskinesis in hook-plated acromioclavicular dislocation including hook motion. J Shoulder Elbow Surg. 2018;27(6):1117-24. 